East Asian Math. J.

Vol. 30 (2014), No. 3, pp. 259-270

http://dx.doi.org/10.7858/eamj.2014.016

\title{
ON GENERALIZED TRIANGULAR MATRIX RINGS
}

\author{
Jang Ho Chun and June Won Park
}

\begin{abstract}
Aвstract. For a generalized triangular matrix ring $T=\left[\begin{array}{cc}R & M \\ 0 & S\end{array}\right]$, over rings $R$ and $S$ having only the idempotents 0 and 1 and over an $(R, S)$-bimodule $M$, we characterize all homomorphisms $\alpha$ 's and all $\alpha$ derivations of $T$. Some of the homomorphisms are compositions of an inner homomorphism and an extended or a twisted homomorphism.
\end{abstract}

\section{Introduction}

For $R$ and $S$ are rings with identity and $M$ is an $(R, S)$-bimodule, we consider a generalized triangular matrix $\operatorname{ring} T=\left[\begin{array}{cc}R & M \\ 0 & S\end{array}\right]$. Automorphisms of $T$ were characterized when $R$ and $S$ have only the trivial idempotents 0 and 1(see[5]). Moreover, in case $R$ and $S$ are strongly indecomposable all automorphisms of $T$ are observed in [1]. In these cases, every automorphism is a composition of an extended automorphism and an inner automorphism. In [4], Ghosseiri determined the structure of $(\alpha, \beta)$-derivations of $T$, where $\alpha$ and $\beta$ are automorphisms of $T$. Moreover, Ghahramani and Moussavi characterized homomorphisms and derivations of $T$ (see [2],[3]).

In this paper, we characterize all homomorphisms $\alpha$ 's of $T$ and all $\alpha$-derivations of $T$, and get four types of homomorphisms of $T$, where one type is a composition of an inner homomorphism and an extended homomorphism and other one type is a composition of an inner homomorphism and a twisted homomorphism.

Throughout this paper, for a generalized triangular matrix ring $T, R$ and $S$ have only the trivial idempotents 0 and 1 and $M$ is an $(R, S)$-bimodule. Every endomorphism $\alpha$ means a ring homomorphism preserving identity i.e., $\alpha(1)=1$ and for a homomorphism $\alpha$ the additive map $\delta: T \rightarrow T$ is called an $\alpha$-derivation if $\delta\left(t t^{\prime}\right)=\alpha(t) \delta\left(t^{\prime}\right)+\delta(t) t^{\prime},\left(t, t^{\prime} \in T\right)$.

Received February 25, 2013; Accepted December 13, 2013

2010 Mathematics Subject Classification. 16S32, 16S50.

Key words and phrases. automorphisms, homomorphisms, derivations, generalized triangular matrix rings.

Thank you for reading.

(C)2014 The Youngnam Mathematical Society (pISSN 1226-6973, eISSN 2287-2833) 


\section{Homomorphisms of $T$}

In this section, we characterize that there exist only four types of homomorphisms of $T$. First we define four types of homomorphisms.

Type I. Let $\phi_{1}: R \rightarrow R$ and $\psi_{1}: S \rightarrow S$ be homomorphisms and $\theta_{1}: M \rightarrow$ $M$ is a $\phi_{1}, \psi_{1}$-bimodule homomorphism. For a fixed element $m$ of $M$, if we define $\alpha: T \rightarrow T$ by

$$
\alpha\left(\left[\begin{array}{ll}
a & b \\
0 & c
\end{array}\right]\right)=\left[\begin{array}{cc}
\phi_{1}(a) & \theta_{1}(b) \\
0 & \psi_{1}(c)
\end{array}\right],\left(\left[\begin{array}{cc}
a & b \\
0 & c
\end{array}\right] \in T\right) .
$$

Then we can easily check that $\alpha$ is a homomorphism of $T$. Since $\left[\begin{array}{cc}1 & -m \\ 0 & 1\end{array}\right]$ is an inverse element of $\left[\begin{array}{cc}1 & m \\ 0 & 1\end{array}\right],\left[\begin{array}{cc}1 & m \\ 0 & 1\end{array}\right]$ induces an inner automorphism $i n n_{m_{t}}$, where $m_{t}$ stands for $\left[\begin{array}{cc}1 & m \\ 0 & 1\end{array}\right]$.

If we define $\alpha_{1}=i n n_{m_{t}} \cdot \alpha$, then $\alpha_{1}$ is a homomorphism i.e.,

$\alpha_{1}\left(\left[\begin{array}{ll}a & b \\ 0 & c\end{array}\right]\right)=\left[\begin{array}{cc}1 & -m \\ 0 & 1\end{array}\right]\left[\begin{array}{cc}\phi_{1}(a) & \theta_{1}(b) \\ 0 & \psi_{1}(c)\end{array}\right]\left[\begin{array}{cc}1 & m \\ 0 & 1\end{array}\right],\left(\left[\begin{array}{cc}a & b \\ 0 & c\end{array}\right] \in T\right)$.

This homomorphism $\alpha_{1}$ is a composition of an inner automorphism and an extended homomorphism.

Type II. Let $\phi_{2}: R \rightarrow S$ and $\psi_{2}: S \rightarrow R$ be homomorphisms. For a fixed element $m$ of $M$, if we define $\alpha_{2}: T \rightarrow T$ by

$\alpha_{2}\left(\left[\begin{array}{ll}a & b \\ 0 & c\end{array}\right]\right)=\left[\begin{array}{cc}1 & m \\ 0 & 1\end{array}\right]\left[\begin{array}{cc}\psi_{2}(c) & 0 \\ 0 & \phi_{2}(a)\end{array}\right]\left[\begin{array}{cc}1 & -m \\ 0 & 1\end{array}\right],\left(\left[\begin{array}{cc}a & b \\ 0 & c\end{array}\right] \in T\right)$.

Then $\alpha_{2}$ is a homomorphism, which is a composition of an inner automorphism and a twisted homomorphism.

Type III. Let $\phi_{3}: R \rightarrow R$ and $\psi_{3}: R \rightarrow S$ be homomorphisms and $\theta_{3}: R \rightarrow$ $M$ be an additive map such that $\theta_{3}\left(a a^{\prime}\right)=\phi_{3}(a) \theta_{3}\left(a^{\prime}\right)+\theta_{3}(a) \psi_{3}\left(a^{\prime}\right)\left(a, a^{\prime} \in R\right)$. If we define $\alpha_{3}: T \rightarrow T$ by

$$
\alpha_{3}\left(\left[\begin{array}{ll}
a & b \\
0 & c
\end{array}\right]\right)=\left[\begin{array}{cc}
\phi_{3}(a) & \theta_{3}(a) \\
0 & \psi_{3}(a)
\end{array}\right],\left(\left[\begin{array}{cc}
a & b \\
0 & c
\end{array}\right] \in T\right) .
$$

Then $\alpha_{3}$ is a homomorphism. 
Type IV. Let $\phi_{4}: S \rightarrow R$ and $\psi_{4}: S \rightarrow S$ be homomorphisms and $\theta_{4}: S \rightarrow M$ be an additive homomorphism such that $\theta_{4}\left(c c^{\prime}\right)=\phi_{4}(c) \theta_{4}\left(c^{\prime}\right)+$ $\theta_{4}(c) \psi_{4}\left(c^{\prime}\right),\left(c, c^{\prime} \in S\right)$. If we define $\alpha_{4}: T \rightarrow T$ by

$$
\alpha_{4}\left(\left[\begin{array}{ll}
a & b \\
0 & c
\end{array}\right]\right)=\left[\begin{array}{cc}
\phi_{4}(c) & \theta_{4}(c) \\
0 & \psi_{4}(c)
\end{array}\right],\left(\left[\begin{array}{ll}
a & b \\
0 & c
\end{array}\right] \in T\right)
$$

Then $\alpha_{4}$ is a homomorphism.

For convenience, denote the idempotents $e_{11}=\left[\begin{array}{ll}1 & 0 \\ 0 & 0\end{array}\right]$ and $e_{22}=\left[\begin{array}{ll}0 & 0 \\ 0 & 1\end{array}\right]$. Also, $0=\left[\begin{array}{ll}0 & 0 \\ 0 & 0\end{array}\right]$ and $1=\left[\begin{array}{ll}1 & 0 \\ 0 & 1\end{array}\right]$.

Lemma 2.1. If $\alpha: T \rightarrow T$ is a homomorphism such that $\alpha\left(e_{11}\right)=$ $\underset{b \in M \text {. Then }}{\left[\begin{array}{cc}r_{1} & m_{1} \\ 0 & s_{1}\end{array}\right], \alpha\left(e_{22}\right)=\left[\begin{array}{cc}r_{2} & m_{2} \\ 0 & s_{2}\end{array}\right] \text { and } \alpha\left(\left[\begin{array}{ll}0 & b \\ 0 & 0\end{array}\right]\right)=\left[\begin{array}{cc}r_{b} & m_{b} \\ 0 & s_{b}\end{array}\right] \text { for }}$

i) $r_{1}, s_{1}, r_{2}, s_{2}$ are 0 or 1 and $r_{i} m_{i}+m_{i} s_{i}=m_{i}(i=1,2)$.

ii) $r_{1}+r_{2}=1, m_{1}+m_{2}=0$ and $s_{1}+s_{2}=1$.

iii) $r_{b}=s_{b}=0, r_{1} m_{b}=m_{b}$ and $m_{b} s_{1}=0$.

Proof.

i) Since $\alpha\left(e_{11}\right)=\left(\alpha\left(e_{11}\right)\right)^{2},\left[\begin{array}{cc}r_{1} & m_{1} \\ 0 & s_{1}\end{array}\right]=\left[\begin{array}{cc}r_{1} & m_{1} \\ 0 & s_{1}\end{array}\right]\left[\begin{array}{cc}r_{1} & m_{1} \\ 0 & s_{1}\end{array}\right]=$ $\left[\begin{array}{cc}r_{1}^{2} & r_{1} m_{1}+m_{1} s_{1} \\ 0 & s_{1}{ }^{2}\end{array}\right]$. Then $r_{1}^{2}=r_{1}, \quad s_{1}^{2}=s_{1}$ and $r_{1} m_{1}+m_{1} s_{1}=m_{1}$. Similarly, $r_{2}{ }^{2}=r_{2}, s_{2}{ }^{2}=s_{2}$ and $r_{2} m_{2}+m_{2} s_{2}=m_{2}$. Thus $r_{1}, s_{1}, r_{2}, s_{2}$ are 0 or 1.

ii) since $1=\alpha(1)=\alpha\left(e_{11}\right)+\alpha\left(e_{22}\right)=\left[\begin{array}{cc}r_{1}+r_{2} & m_{1}+m_{2} \\ 0 & s_{1}+s_{2}\end{array}\right], r_{1}+r_{2}=$ $1, m_{1}+m_{2}=0$ and $s_{1}+s_{2}=1$.

iii) By hypothesis, $\alpha\left(\left[\begin{array}{ll}0 & b \\ 0 & 0\end{array}\right]\right)=\left[\begin{array}{cc}r_{b} & m_{b} \\ 0 & s_{b}\end{array}\right]$ and $\alpha\left(\left[\begin{array}{ll}0 & b \\ 0 & 0\end{array}\right]\right)=$ $\alpha\left(e_{11}\right) \alpha\left(\left[\begin{array}{ll}0 & b \\ 0 & 0\end{array}\right]\right)=\left[\begin{array}{cc}r_{1} & m_{1} \\ 0 & s_{1}\end{array}\right]\left[\begin{array}{cc}r_{b} & m_{b} \\ 0 & s_{b}\end{array}\right]=\left[\begin{array}{cc}r_{1} r_{b} & r_{1} m_{b}+m_{1} s_{b} \\ 0 & s_{1} s_{b}\end{array}\right]$. So, $r_{1} r_{b}=r_{b}, s_{1} s_{b}=s_{b}$ and $r_{1} m_{b}+m_{1} s_{b}=m_{b}$.

On the other hand, $0=\alpha(0)=\alpha\left(\left[\begin{array}{ll}0 & b \\ 0 & 0\end{array}\right]\right) \alpha\left(e_{11}\right)=\left[\begin{array}{cc}r_{b} & m_{b} \\ 0 & s_{b}\end{array}\right]$ $\left[\begin{array}{cc}r_{1} & m_{1} \\ 0 & s_{1}\end{array}\right]=\left[\begin{array}{cc}r_{b} r_{1} & r_{b} m_{1}+m_{b} s_{1} \\ 0 & s_{b} s_{1}\end{array}\right]$. So, $r_{b} r_{1}=0, s_{b} s_{1}=0$ and $r_{b} m_{1}+$ $m_{b} s_{1}=0$. Thus $r_{b}=s_{b}=0, r_{1} m_{b}=m_{b}$ and $m_{b} s_{1}=0$. 
Remark 2.2. From Lemma 2.1, we conclude that there are only four cases of homomorphisms $\alpha$ 's which are depended on the values of $r_{i}, s_{i}, m_{i}$. The four cases are followings ;

Case I. $r_{1}=1, r_{2}=0, s_{1}=0, s_{2}=1$. This implies $m_{1}=m, m_{2}=-m$.

Case II. $r_{1}=0, r_{2}=1, s_{1}=1, s_{2}=0$. This implies $m_{1}=m, m_{2}=-m$ and $m_{b}=0$.

Case III. $r_{1}=1, r_{2}=0, s_{1}=1, s_{2}=0$. This implies $m_{1}=m_{2}=m_{b}=0$.

Case IV. $r_{1}=0, r_{2}=1, s_{1}=0, s_{2}=1$. This implies $m_{1}=m_{2}=m_{b}=0$.

From Lemma 2.1 and Remark 2.2, we have the following theorems.

Theorem 2.3. Case I homomorphisms are Type I homomorphisms.

Proof. Since $r_{1}=s_{2}=1, r_{2}=s_{1}=0, \alpha\left(e_{11}\right)=\left[\begin{array}{cc}1 & m \\ 0 & 0\end{array}\right], \alpha\left(e_{22}\right)=$ $\left[\begin{array}{cc}0 & -m \\ 0 & 1\end{array}\right]$ and $\alpha\left(\left[\begin{array}{ll}0 & b \\ 0 & 0\end{array}\right]\right)=\left[\begin{array}{cc}0 & m_{b} \\ 0 & 0\end{array}\right]$. Thus we can define $\theta_{1}: M \rightarrow$ $M$ by $\theta_{1}(b)=m_{b}$.

Let $\alpha\left(a e_{11}\right)=\left(\left[\begin{array}{cc}r_{a} & m_{a} \\ 0 & s_{a}\end{array}\right]\right)$. Then $\alpha\left(a e_{11}\right)=\alpha\left(a e_{11}\right) \alpha\left(e_{11}\right)=\left[\begin{array}{cc}r_{a} & m_{a} \\ 0 & s_{a}\end{array}\right]$ $\left[\begin{array}{cc}1 & m \\ 0 & 0\end{array}\right]=\left[\begin{array}{cc}r_{a} & r_{a} m \\ 0 & 0\end{array}\right]$. So, $s_{a}=0$ and $m_{a}=r_{a} m$. Thus we can define $\phi_{1}: R \rightarrow R$ by $\phi_{1}(a)=r_{a}$.

Let $\alpha\left(c e_{22}\right)=\left(\left[\begin{array}{cc}r_{c} & m_{c} \\ 0 & s_{c}\end{array}\right]\right)$. Then $\left.\alpha\left(c e_{22}\right)=\alpha\left(e_{22}\right) \alpha\left(c e_{22}\right)\right]=\left[\begin{array}{cc}0 & -m \\ 0 & 1\end{array}\right]$ $\left[\begin{array}{cc}r_{c} & m_{c} \\ 0 & s_{c}\end{array}\right]=\left[\begin{array}{cc}0 & -m s_{c} \\ 0 & s_{c}\end{array}\right]$. So, $r_{c}=0$ and $m_{c}=-m s_{c}$. Thus we can define $\psi_{1}: S \rightarrow S$ by $\psi_{1}(c)=s_{c}$.

Therefore, we conclude $\alpha\left(\left[\begin{array}{ll}a & b \\ 0 & c\end{array}\right]\right)=\left[\begin{array}{cc}r_{a} & r_{a} m+m_{b}-m s_{c} \\ 0 & s_{c}\end{array}\right]=$ $\left[\begin{array}{cc}\phi_{1}(a) & \phi_{1}(a) m+\theta_{1}(b)-m \psi_{1}(c) \\ 0 & \psi_{1}(c)\end{array}\right]=\left[\begin{array}{cc}1 & -m \\ 0 & 1\end{array}\right]\left[\begin{array}{cc}\phi_{1}(a) & \theta_{1}(b) \\ 0 & \psi_{1}(c)\end{array}\right]\left[\begin{array}{cc}1 & m \\ 0 & 1\end{array}\right]$.

Moreover, for $a \in R$ and $b \in M, \alpha\left(\left[\begin{array}{cc}0 & a b \\ 0 & 0\end{array}\right]\right)=\alpha\left(a e_{11}\right) \alpha\left(\left[\begin{array}{ll}0 & b \\ 0 & 0\end{array}\right]\right)=$ $\left[\begin{array}{cc}\phi_{1}(a) & \phi_{1}(a) m \\ 0 & 0\end{array}\right]\left[\begin{array}{cc}0 & \theta_{1}(b) \\ 0 & 0\end{array}\right]=\left[\begin{array}{cc}0 & \phi_{1}(a) \theta_{1}(b) \\ 0 & 0\end{array}\right]$ and $\alpha\left[\begin{array}{cc}0 & a b \\ 0 & 0\end{array}\right]=$ $\left[\begin{array}{cc}0 & \theta_{1}(a b) \\ 0 & 0\end{array}\right]$. So, $\theta_{1}(a b)=\phi_{1}(a) \theta_{1}(b)$.

Similarly, for $b \in M$ and $c \in S, \theta_{1}(b c)=\theta_{1}(b) \psi_{1}(c)$.

Theorem 2.4. Case II homomorphisms are Type II homomorphisms.

Proof. Since $r_{1}=s_{2}=0, r_{2}=s_{1}=1$ and $r_{b}=s_{b}=m_{b}=0, \alpha\left(e_{11}\right)=$ $\left[\begin{array}{cc}0 & m \\ 0 & 1\end{array}\right], \alpha\left(e_{22}\right)=\left[\begin{array}{cc}1 & -m \\ 0 & 0\end{array}\right]$ and $\alpha\left(\left[\begin{array}{ll}0 & b \\ 0 & 0\end{array}\right]\right)=0$. 
Let $\alpha\left(a e_{11}\right)=\left[\begin{array}{cc}r_{a} & m_{a} \\ 0 & s_{a}\end{array}\right]$. Then $\alpha\left(a e_{11}\right)=\alpha\left(e_{11}\right) \alpha\left(a e_{11}\right)=\left[\begin{array}{cc}0 & m \\ 0 & 1\end{array}\right]$ $\left[\begin{array}{cc}r_{a} & m_{a} \\ 0 & s_{a}\end{array}\right]=\left[\begin{array}{cc}0 & m s_{a} \\ 0 & s_{a}\end{array}\right]$. So, $r_{a}=0$. Thus we can define $\phi_{2}: R \rightarrow S$ by $\phi_{2}(a)=s_{a}$.

Let $\left.\alpha\left(c e_{22}\right)\right)=\left[\begin{array}{cc}r_{c} & m_{c} \\ 0 & s_{c}\end{array}\right]$. Then $\alpha\left(c e_{22}\right)=\alpha\left(c e_{22}\right) \alpha\left(e_{22}\right)=\left[\begin{array}{cc}r_{c} & m_{c} \\ 0 & s_{c}\end{array}\right]$ $\left[\begin{array}{cc}1 & -m \\ 0 & 0\end{array}\right]=\left[\begin{array}{cc}r_{c} & -r_{c} m \\ 0 & 0\end{array}\right]$. So, $s_{c}=0$. Thus we can define $\psi_{2}: S \rightarrow R$ by $\psi_{2}(c)=r_{c}$.

Therefore, we conclude $\alpha\left(\left[\begin{array}{cc}a & b \\ 0 & c\end{array}\right]\right)=\left[\begin{array}{cc}r_{c} & m s_{a}-r_{c} m \\ 0 & s_{a}\end{array}\right]$ $=\left[\begin{array}{cc}\psi_{2}(c) & m \phi_{2}(a)-\psi_{2}(c) m \\ 0 & \phi_{2}(a)\end{array}\right]=\left[\begin{array}{cc}1 & m \\ 0 & 1\end{array}\right]\left[\begin{array}{cc}\psi_{2}(c) & 0 \\ 0 & \phi_{2}(a)\end{array}\right]\left[\begin{array}{cc}1 & -m \\ 0 & 1\end{array}\right]$.

Theorem 2.5. Case III homomorphisms are Type III homomorphisms.

Proof. Since $r_{1}=s_{1}=1, r_{2}=s_{2}=0$ and $m_{1}=m_{2}=m_{b}=0, \alpha\left(e_{11}\right)=$ $\left[\begin{array}{ll}1 & 0 \\ 0 & 1\end{array}\right], \alpha\left(e_{22}\right)=0$ and $\alpha\left(\left[\begin{array}{ll}0 & b \\ 0 & 0\end{array}\right]\right)=0$

Let $\alpha\left(a e_{11}\right)=\left[\begin{array}{cc}r_{a} & m_{a} \\ 0 & s_{a}\end{array}\right]$, then we can define $\phi_{3}: R \rightarrow R$ by $\phi_{3}(a)=$ $r_{a}, \psi_{3}: R \rightarrow S$ by $\psi_{3}(a)=s_{a}$ and $\theta_{3}: R \rightarrow M$ by $\theta_{3}(a)=m_{a}$.

Now, for $a, a^{\prime} \in R, \alpha\left(a a^{\prime} e_{11}\right)=\alpha\left(a e_{11}\right) \alpha\left(a^{\prime} e_{11}\right)=\left[\begin{array}{cc}\phi_{3}(a) & \theta_{3}(a) \\ 0 & \psi_{3}(a)\end{array}\right]$ $\left[\begin{array}{cc}\phi_{3}\left(a^{\prime}\right) & \theta_{3}\left(a^{\prime}\right) \\ 0 & \psi_{3}\left(a^{\prime}\right)\end{array}\right]=\left[\begin{array}{cc}\phi_{3}(a) \phi_{3}\left(a^{\prime}\right) & \phi_{3}(a) \theta_{3}\left(a^{\prime}\right)+\theta_{3}(a) \psi_{3}\left(a^{\prime}\right) \\ 0 & \psi_{3}(a) \psi_{3}\left(a^{\prime}\right)\end{array}\right]$ and $\alpha\left(a a^{\prime} e_{11}\right)$ $=\left[\begin{array}{cc}\phi_{3}\left(a a^{\prime}\right) & \theta_{3}\left(a a^{\prime}\right) \\ 0 & \psi_{3}\left(a a^{\prime}\right)\end{array}\right]$. This means that $\phi_{3}, \psi_{3}$ are homomorphisms and $\theta_{3}$ is additive, which satisfies $\theta_{3}\left(a a^{\prime}\right)=\phi_{3}(a) \theta_{3}\left(a^{\prime}\right)+\theta_{3}(a) \psi_{3}\left(a^{\prime}\right)$.

Theorem 2.6. Case IV homomorphisms are Type IV homomorphisms. Proof. The proof is similar to the proof of Theorem 2.5.

Remark 2.7. Case II, III and IV homomorphisms cannot be isomorphisms. So, every automorphism of $T$ is a Type I automorphism which is a composition of an inner automorphism and an extended automorphism. 


\section{Derivations of $T$}

In this section, we will observe all $\alpha$-derivations where $\alpha$ is a homomorphism given in Section 2. Since there are four types of homomorphisms, we get four types of $\alpha$-derivations.

Theorem 3.1. Let $\alpha_{1}: T \rightarrow T$ be a Type I homomorphism for some fixed element $m \in M$. Then $\delta: T \rightarrow T$ is an $\alpha_{1}$-derivation if and only if there exist

(i) $f: R \rightarrow R$ is a $\phi_{1}$-derivation,

(ii) $g: S \rightarrow S$ is a $\psi_{1}$-derivation and

(iii) $h: M \rightarrow M$ is an additive map satisfying $h(a b)=\phi_{1}(a) h(b)+f(a) b$ and $h(b c)=\theta_{1}(b) g(c)+h(b) c$ such that for some $b_{1} \in M$,

$$
\delta\left(\left[\begin{array}{ll}
a & b \\
0 & c
\end{array}\right]\right)=\left[\begin{array}{cc}
f(a) & \phi_{1}(a) b_{1}-b_{1} c-m g(c)+h(b) \\
0 & g(c)
\end{array}\right]
$$

where $\phi_{1}, \psi_{1}, \theta_{1}$ are maps given in Type I homomorphisms in Section 2.

Proof. Since $\alpha_{1}$ is a Type I homomorphism, assume $\alpha_{1}\left(\left[\begin{array}{ll}a & b \\ 0 & c\end{array}\right]\right)=$ $\left[\begin{array}{cc}1 & -m \\ 0 & 1\end{array}\right]\left[\begin{array}{cc}\phi_{1}(a) & \theta_{1}(b) \\ 0 & \psi_{1}(c)\end{array}\right]\left[\begin{array}{cc}1 & m \\ 0 & 1\end{array}\right]=\left[\begin{array}{cc}\phi_{1}(a) & \phi_{1}(a) m+\theta_{1}(b)-m \psi_{1}(c) \\ 0 & \psi_{1}(c)\end{array}\right]$ for some $m \in M$.

Let $\delta\left(e_{11}\right)=\left[\begin{array}{cc}a_{1} & b_{1} \\ 0 & c_{1}\end{array}\right]$. Then $\delta\left(e_{11}\right)=\alpha_{1}\left(e_{11}\right) \delta\left(e_{11}\right)+\delta\left(e_{11}\right) e_{11}=\left[\begin{array}{cc}1 & m \\ 0 & 0\end{array}\right]$ $\left[\begin{array}{cc}a_{1} & b_{1} \\ 0 & c_{1}\end{array}\right]+\left[\begin{array}{cc}a_{1} & b_{1} \\ 0 & c_{1}\end{array}\right] e_{11}=\left[\begin{array}{cc}a_{1} & b_{1}+m c_{1} \\ 0 & 0\end{array}\right]+a_{1} e_{11}=\left[\begin{array}{cc}2 a_{1} & b_{1}+m c_{1} \\ 0 & 0\end{array}\right]$. So, $c_{1}=a_{1}=0$. Thus $\delta\left(e_{11}\right)=\left[\begin{array}{cc}0 & b_{1} \\ 0 & 0\end{array}\right]$.

Since $0=\delta(1)=\delta\left(e_{11}\right)+\delta\left(e_{22}\right), \delta\left(e_{22}\right)=\left[\begin{array}{cc}0 & -b_{1} \\ 0 & 0\end{array}\right]$.

(i) Let $\delta\left(a e_{11}\right)=\left[\begin{array}{cc}a_{R} & a_{M} \\ 0 & a_{S}\end{array}\right]$. Then $\delta\left(a e_{11}\right)=\delta\left(e_{11} a e_{11}\right)=\alpha_{1}\left(e_{11}\right) \delta\left(a e_{11}\right)+$ $\delta\left(e_{11}\right)\left(a e_{11}\right)=\left[\begin{array}{cc}1 & m \\ 0 & 0\end{array}\right]\left[\begin{array}{cc}a_{R} & a_{M} \\ 0 & a_{S}\end{array}\right]+\left[\begin{array}{cc}0 & b_{1} \\ 0 & 0\end{array}\right] a e_{11}=\left[\begin{array}{cc}a_{R} & a_{M}+m a_{S} \\ 0 & 0\end{array}\right]$. So, $a_{S}=0$. Thus $\delta\left(a e_{11}\right)=\left[\begin{array}{cc}a_{R} & a_{M} \\ 0 & 0\end{array}\right]$.

On the other hand, $\delta\left(a e_{11}\right)=\delta\left(a e_{11} e_{11}\right)=\alpha_{1}\left(a e_{11}\right) \delta\left(e_{11}\right)+\delta\left(a e_{11}\right) e_{11}=$ $\left[\begin{array}{cc}\phi_{1}(a) & \phi_{1}(a) m \\ 0 & 0\end{array}\right]\left[\begin{array}{cc}0 & b_{1} \\ 0 & 0\end{array}\right]+\left[\begin{array}{cc}a_{R} & a_{M} \\ 0 & 0\end{array}\right] e_{11}=\left[\begin{array}{cc}0 & \phi_{1}(a) b_{1} \\ 0 & 0\end{array}\right]+a_{R} e_{11}=$ $\left[\begin{array}{cc}a_{R} & \phi_{1}(a) b_{1} \\ 0 & 0\end{array}\right]$. So, $a_{M}=\phi_{1}(a) b_{1}$. Thus $\delta\left(a e_{11}\right)=\left[\begin{array}{cc}a_{R} & \phi_{1}(a) b_{1} \\ 0 & 0\end{array}\right]$.

If we define $f: R \rightarrow R$ by $f(a)=a_{R}$, then we can easily check that $f$ is a $\phi_{1}$-derivation. 
(ii) Let $\delta\left(c e_{22}\right)=\left[\begin{array}{cc}c_{R} & c_{M} \\ 0 & c_{S}\end{array}\right]$. Then $\delta\left(c e_{22}\right)=\delta\left(e_{22} c e_{22}\right)=\alpha_{1}\left(e_{22}\right) \delta\left(c e_{22}\right)+$ $\delta\left(e_{22}\right) c e_{22}=\left[\begin{array}{cc}0 & -m \\ 0 & 1\end{array}\right]\left[\begin{array}{cc}c_{R} & c_{M} \\ 0 & c_{S}\end{array}\right]+\left[\begin{array}{cc}0 & -b_{1} \\ 0 & 0\end{array}\right] c e_{22}=\left[\begin{array}{cc}0 & -m c_{S} \\ 0 & c_{S}\end{array}\right]+$ $\left[\begin{array}{cc}0 & -b_{1} c \\ 0 & 0\end{array}\right]=\left[\begin{array}{cc}0 & -m c_{S}-b_{1} c \\ 0 & c_{S}\end{array}\right]$. So, $c_{R}=0$ and $-b_{1} c-m c_{S}=c_{M}$. Thus $\delta\left(c e_{22}\right)=\left[\begin{array}{cc}0 & -b_{1} c-m c_{S} \\ 0 & c_{S}\end{array}\right]$.

If we define $g: S \rightarrow S$ by $g(c)=c_{S}$, then $g$ is a $\psi_{1}$-derivation.

(iii) Let $\delta\left(\left[\begin{array}{ll}0 & b \\ 0 & 0\end{array}\right]\right)=\left[\begin{array}{cc}b_{R} & b_{M} \\ 0 & b_{S}\end{array}\right]$. Then $\delta\left(\left[\begin{array}{ll}0 & b \\ 0 & 0\end{array}\right]\right)=\delta\left(e_{11}\left[\begin{array}{ll}0 & b \\ 0 & 0\end{array}\right]\right)$ $=\alpha_{1}\left(e_{11}\right) \delta\left(\left[\begin{array}{ll}0 & b \\ 0 & 0\end{array}\right]\right)+\delta\left(e_{11}\right)\left[\begin{array}{cc}0 & b \\ 0 & 0\end{array}\right]=\left[\begin{array}{cc}1 & m \\ 0 & 0\end{array}\right]\left[\begin{array}{cc}b_{R} & b_{M} \\ 0 & b_{S}\end{array}\right]+\left[\begin{array}{cc}0 & b_{1} \\ 0 & 0\end{array}\right]$ $\left[\begin{array}{ll}0 & b \\ 0 & 0\end{array}\right]=\left[\begin{array}{cc}b_{R} & b_{M}+m b_{S} \\ 0 & 0\end{array}\right]$. So, $b_{S}=0$.

On the other hand, $\delta\left(\left[\begin{array}{ll}0 & b \\ 0 & 0\end{array}\right]\right)=\delta\left(\left[\begin{array}{ll}0 & b \\ 0 & 0\end{array}\right] e_{22}\right)=\alpha_{1}\left(\left[\begin{array}{ll}0 & b \\ 0 & 0\end{array}\right]\right) \delta\left(e_{22}\right)+$ $\delta\left(\left[\begin{array}{ll}0 & b \\ 0 & 0\end{array}\right]\right) e_{22}=\left[\begin{array}{cc}0 & \theta_{1}(b) \\ 0 & 0\end{array}\right]\left[\begin{array}{cc}0 & -b_{1} \\ 0 & 0\end{array}\right]+\left[\begin{array}{cc}b_{R} & b_{M} \\ 0 & b_{S}\end{array}\right] e_{22}=\left[\begin{array}{cc}0 & b_{M} \\ 0 & b_{S}\end{array}\right]$. So, $b_{R}=0$. Thus $\delta\left(\left[\begin{array}{ll}0 & b \\ 0 & 0\end{array}\right]\right)=\left[\begin{array}{cc}0 & b_{M} \\ 0 & 0\end{array}\right]$.

If we define $h: M \rightarrow M$ by $h(b)=b_{M}$, then $h$ satisfies the property of (iii).

This means $\delta\left(a e_{11}\right)=\left[\begin{array}{cc}f(a) & \phi_{1}(a) b_{1} \\ 0 & 0\end{array}\right], \delta\left(c e_{22}\right)=\left[\begin{array}{cc}0 & -b_{1} c-m g(c) \\ 0 & g(c)\end{array}\right]$, and $\delta\left(\left[\begin{array}{ll}0 & b \\ 0 & 0\end{array}\right]\right)=\left[\begin{array}{cc}0 & b_{M} \\ 0 & 0\end{array}\right]$. That is

$$
\delta\left(\left[\begin{array}{ll}
a & b \\
0 & c
\end{array}\right]\right)=\left[\begin{array}{cc}
f(a) & \phi_{1}(a) b_{1}-b_{1} c-m g(c)+h(b) \\
0 & g(c)
\end{array}\right] .
$$

Conversely, we define $\delta\left(\left[\begin{array}{cc}a & b \\ 0 & c\end{array}\right]\right)=\left[\begin{array}{cc}f(a) & \phi_{1}(a) b_{1}-b_{1} c-m g(c)+h(b) \\ 0 & g(c)\end{array}\right]$. Then $\alpha_{1}\left(\left[\begin{array}{ll}a & b \\ 0 & c\end{array}\right]\right) \delta\left(\left[\begin{array}{cc}a^{\prime} & b^{\prime} \\ 0 & c^{\prime}\end{array}\right]\right)+\delta\left(\left[\begin{array}{ll}a & b \\ 0 & c\end{array}\right]\right)\left[\begin{array}{ll}a^{\prime} & b^{\prime} \\ 0 & c^{\prime}\end{array}\right]$ $=\left[\begin{array}{cc}\phi_{1}(a) & \phi_{1}(a) m+\theta_{1}(b)-m \psi_{1}(c) \\ 0 & \psi_{1}(c)\end{array}\right]\left[\begin{array}{cc}f\left(a^{\prime}\right) & \phi_{1}\left(a^{\prime}\right) b_{1}-b_{1} c^{\prime}-m g\left(c^{\prime}\right)+h\left(b^{\prime}\right) \\ 0 & g\left(c^{\prime}\right)\end{array}\right]$ $+\left[\begin{array}{cc}f(a) & \phi_{1}(a) b_{1}-b_{1} c-m g(c)+h(b) \\ 0 & g(c)\end{array}\right]\left[\begin{array}{cc}a^{\prime} & b^{\prime} \\ 0 & c^{\prime}\end{array}\right]$ $=\left[\begin{array}{cc}\phi_{1}(a) f\left(a^{\prime}\right) & A \\ 0 & \psi_{1}(c) g\left(c^{\prime}\right)\end{array}\right]+\left[\begin{array}{cc}f(a) a^{\prime} & A^{\prime} \\ 0 & g(c) c^{\prime}\end{array}\right]$, where $A=\phi_{1}(a) \phi_{1}\left(a^{\prime}\right) b_{1}-$ $\phi_{1}(a) b_{1} c^{\prime}-\phi_{1}(a) m g\left(c^{\prime}\right)+\phi_{1}(a) h\left(b^{\prime}\right)+\phi_{1}(a) m g\left(c^{\prime}\right)+\theta_{1}(b) g\left(c^{\prime}\right)-m \psi_{1}(c) g\left(c^{\prime}\right)$ and $A^{\prime}=f(a) b^{\prime}+\phi_{1}(a) b_{1} c^{\prime}-b_{1} c c^{\prime}-m g(c) c^{\prime}+h(b) c^{\prime}$.

Here, $(1,1)$-component is $\phi_{1}(a) f\left(a^{\prime}\right)+f(a) a^{\prime}=f\left(a a^{\prime}\right)$. 
$(2,2)$ - component is $\psi_{1}(c) g\left(c^{\prime}\right)+g(c) c^{\prime}=g\left(c c^{\prime}\right)$.

$(1,2)$-component is $\phi_{1}(a) \phi_{1}\left(a^{\prime}\right) b_{1}-\phi_{1}(a) b_{1} c^{\prime}-\phi_{1}(a) m g\left(c^{\prime}\right)+\phi_{1}(a) h\left(b^{\prime}\right)+$ $\phi_{1}(a) m g\left(c^{\prime}\right)+\theta_{1}(b) g\left(c^{\prime}\right)-m \psi_{1}(c) g\left(c^{\prime}\right)+f(a) b^{\prime}+\phi_{1}(a) b_{1} c^{\prime}-b_{1} c c^{\prime}-m g(c) c^{\prime}+$ $h(b) c^{\prime}=\phi_{1}\left(a a^{\prime}\right) b_{1}-b_{1} c c^{\prime}-m g\left(c c^{\prime}\right)+h\left(a b^{\prime}+b c^{\prime}\right)$.

On the other hand, $\delta\left(\left[\begin{array}{ll}a & b \\ 0 & c\end{array}\right]\left[\begin{array}{cc}a^{\prime} & b^{\prime} \\ 0 & c^{\prime}\end{array}\right]\right)=\delta\left[\begin{array}{cc}a a^{\prime} & a b^{\prime}+b c^{\prime} \\ 0 & c c^{\prime}\end{array}\right]=$ $\left[\begin{array}{cc}f\left(a a^{\prime}\right) & \phi_{1}\left(a a^{\prime}\right) b_{1}-b_{1} c c^{\prime}-m g\left(c c^{\prime}\right)+h\left(a b^{\prime}+b c^{\prime}\right) \\ 0 & g\left(c c^{\prime}\right)\end{array}\right]$.

Therefore $\delta$ is an $\alpha_{1}$-derivation.

Corollary 3.2. In Theorem 3.1, let $\delta_{1}: T \rightarrow T$ by $\delta_{1}\left(\left[\begin{array}{ll}a & b \\ 0 & c\end{array}\right]\right)=$ $\left[\begin{array}{cc}0 & \phi_{1}(a) b_{1}-b_{1} c \\ 0 & 0\end{array}\right]$ and $\delta_{2}: T \rightarrow T$ by $\delta_{2}\left(\left[\begin{array}{cc}a & b \\ 0 & c\end{array}\right]\right)=\left[\begin{array}{cc}f(a) & -m g(c)+h(b) \\ 0 & g(c)\end{array}\right]$. Then $\delta_{1}$ and $\delta_{2}$ are $\alpha_{1}$-derivations.

Remark 3.3. In Corollary 3.2, $\delta_{1}\left(\left[\begin{array}{ll}a & b \\ 0 & c\end{array}\right]\right)=\left[\begin{array}{cc}0 & \phi_{1}(a) b_{1}-b_{1} c \\ 0 & 0\end{array}\right]=$ $\alpha_{1}\left(\left[\begin{array}{ll}a & b \\ 0 & c\end{array}\right]\right)\left[\begin{array}{cc}0 & b_{1} \\ 0 & 0\end{array}\right]-\left[\begin{array}{cc}0 & b_{1} \\ 0 & 0\end{array}\right]\left[\begin{array}{cc}a & b \\ 0 & c\end{array}\right]$. So, $\delta_{1}$ is an inner $\alpha_{1}$-derivation for $\left[\begin{array}{cc}0 & b_{1} \\ 0 & 0\end{array}\right]$.

Moreover, if $m=0$, then $\alpha_{1}$ is a trivial extension on $T$ and every derivation is a sum of an inner $\alpha_{1}$-derivation and an extended derivation. This satisfies [2, Proposition 2.6] and [3, Theorem 3.2].

Theorem 3.4. Let $\alpha_{2}: T \rightarrow T$ be a Type II homomorphism for some fixed element $m \in M$. Then $\delta: T \rightarrow T$ is an $\alpha_{2}$-derivation if and only if there exist

(i) $g: M \rightarrow S$ is an additive map satisfying $\forall a \in R, b \in M, c \in S, g(a b)=$ $\phi_{2}(a) g(b)$ and $g(b c)=g(b) c$ and

(ii) $h: S \rightarrow M$ is an additive map satisfying $\forall c, s \in S, h(c s)=\psi_{2}(c) h(s)+$ $h(c) s+\psi_{2}(c) m c_{1} s$ for some $c_{1} \in S$ such that for some $a_{1} \in R$,

$$
\delta\left(\left[\begin{array}{ll}
a & b \\
0 & c
\end{array}\right]\right)=\left[\begin{array}{cc}
a_{1} a-\psi_{2}(c) a_{1} & m \phi_{2}(a) c_{1}+a_{1} b+m g(b)+h(c) \\
0 & \phi_{2}(a) c_{1}+g(b)-c_{1} c
\end{array}\right],
$$

where $\phi_{2}, \psi_{2}, \theta_{2}$ are maps given in Type II homomorphisms in Section 2.

Proof. Assume $\delta$ is an $\alpha_{2}$-derivation and $\alpha_{2}$ be Type II homomorphism. So, $\alpha_{2}\left(\left[\begin{array}{ll}a & b \\ 0 & c\end{array}\right]\right)=\left[\begin{array}{cc}1 & m \\ 0 & 1\end{array}\right]\left[\begin{array}{cc}\psi_{2}(c) & 0 \\ 0 & \phi_{2}(a)\end{array}\right]\left[\begin{array}{cc}1 & -m \\ 0 & 1\end{array}\right]=$ $\left[\begin{array}{cc}\psi_{2}(c) & m \phi_{2}(a)-\psi_{2}(c) m \\ 0 & \phi_{2}(a)\end{array}\right]$ for some $m \in M$. 
Let $\delta\left(e_{11}\right)=\left[\begin{array}{cc}a_{1} & b_{1} \\ 0 & c_{1}\end{array}\right]$. Then $\delta\left(e_{11}\right)=\delta\left(e_{11} e_{11}\right)=\alpha_{2}\left(e_{11}\right) \delta\left(e_{11}\right)+\delta\left(e_{11}\right) e_{11}=$ $\left[\begin{array}{cc}0 & m \\ 0 & 1\end{array}\right]\left[\begin{array}{cc}a_{1} & b_{1} \\ 0 & c_{1}\end{array}\right]+\left[\begin{array}{cc}a_{1} & b_{1} \\ 0 & c_{1}\end{array}\right] e_{11}=\left[\begin{array}{cc}a_{1} & m c_{1} \\ 0 & c_{1}\end{array}\right]$. Thus $\delta\left(e_{11}\right)=\left[\begin{array}{cc}a_{1} & m c_{1} \\ 0 & c_{1}\end{array}\right]$ and $\delta\left(e_{22}\right)=\left[\begin{array}{cc}-a_{1} & -m c_{1} \\ 0 & -c_{1}\end{array}\right]$.

Let $\delta\left(\left[\begin{array}{ll}0 & b \\ 0 & 0\end{array}\right]\right)=\left[\begin{array}{cc}b_{R} & b_{M} \\ 0 & b_{S}\end{array}\right]$. Then $\delta\left(\left[\begin{array}{ll}0 & b \\ 0 & 0\end{array}\right]\right)=\delta\left(\left[\begin{array}{ll}0 & b \\ 0 & 0\end{array}\right] e_{22}\right)$ $=\alpha_{2}\left(\left[\begin{array}{ll}0 & b \\ 0 & 0\end{array}\right]\right) \delta\left(e_{22}\right)+\delta\left(\left[\begin{array}{ll}0 & b \\ 0 & 0\end{array}\right]\right) e_{22}=0\left[\begin{array}{cc}-a_{1} & -m c_{1} \\ 0 & -c_{1}\end{array}\right]+\left[\begin{array}{cc}b_{R} & b_{M} \\ 0 & b_{S}\end{array}\right] e_{22}$ $=\left[\begin{array}{cc}0 & b_{M} \\ 0 & b_{S}\end{array}\right]$. So, $b_{R}=0$.

On the other hand, $\delta\left(\left[\begin{array}{ll}0 & b \\ 0 & 0\end{array}\right]\right)=\delta\left(e_{11}\left[\begin{array}{ll}0 & b \\ 0 & 0\end{array}\right]\right)=\alpha_{2}\left(e_{11}\right) \delta\left(\left[\begin{array}{ll}0 & b \\ 0 & 0\end{array}\right]\right)+$ $\delta\left(e_{11}\right)\left[\begin{array}{ll}0 & b \\ 0 & 0\end{array}\right]=\left[\begin{array}{cc}0 & m b_{S} \\ 0 & b_{S}\end{array}\right]+\left[\begin{array}{cc}0 & a_{1} b \\ 0 & 0\end{array}\right]=\left[\begin{array}{cc}0 & m b_{S}+a_{1} b \\ 0 & b_{S}\end{array}\right]$. So, $b_{M}=$ $a_{1} b+m b_{S}$.

Therefore we can define $g: M \rightarrow S$ by $g(b)=b_{S}$, where $\delta\left(\left[\begin{array}{ll}0 & b \\ 0 & 0\end{array}\right]\right)=$ $\left[\begin{array}{cc}0 & a_{1} b+m b_{S} \\ 0 & b_{S}\end{array}\right]$

Let $\delta\left(a e_{11}\right)=\left[\begin{array}{cc}a_{R} & a_{M} \\ 0 & a_{S}\end{array}\right]$. Then $\left.\delta\left(a e_{11}\right)=\delta\left(a e_{11} e_{11}\right)\right)=\alpha_{2}\left(a e_{11}\right) \delta\left(e_{11}\right)+$ $\delta\left(a e_{11}\right) e_{11}=\left[\begin{array}{cc}0 & m s_{a} \\ 0 & s_{a}\end{array}\right]\left[\begin{array}{cc}a_{1} & m c_{1} \\ 0 & c_{1}\end{array}\right]+\left[\begin{array}{cc}a_{R} & a_{M} \\ 0 & a_{S}\end{array}\right] e_{11}=\left[\begin{array}{cc}0 & m s_{a} c_{1} \\ 0 & s_{a} c_{1}\end{array}\right]+$ $a_{R} e_{11}=\left[\begin{array}{cc}a_{R} & m s_{a} c_{1} \\ 0 & s_{a} c_{1}\end{array}\right]=\left[\begin{array}{cc}a_{R} & m \phi_{2}(a) c_{1} \\ 0 & \phi_{2}(a) c_{1}\end{array}\right]$. Thus $a_{S}=\phi_{2}(a) c_{1}$ and $a_{M}=$ $m \phi_{2}(a) c_{1}$.

On the other hand, $\delta\left(a e_{11}\right)=\delta\left(e_{11} a e_{11}\right)$ implies $a_{R}=a_{1} a$. Thus $\delta\left(a e_{11}\right)=$ $\left[\begin{array}{cc}a_{1} a & m \phi_{2}(a) c_{1} \\ 0 & \phi_{2}(a) c_{1}\end{array}\right]$

Let $\delta\left(c e_{22}\right)=\left[\begin{array}{cc}c_{R} & c_{M} \\ 0 & c_{S}\end{array}\right]$. Then $\delta\left(c e_{22}\right)=\delta\left(c e_{22} e_{22}\right)=\alpha_{2}\left(c e_{22}\right) \delta\left(e_{22}\right)+$ $\delta\left(c e_{22}\right) e_{22}=\left[\begin{array}{cc}r_{c} & -r_{c} m \\ 0 & 0\end{array}\right]\left[\begin{array}{cc}-a_{1} & -m c_{1} \\ 0 & -c_{1}\end{array}\right]+\left[\begin{array}{cc}c_{R} & c_{M} \\ 0 & c_{S}\end{array}\right] e_{22}$ $=\left[\begin{array}{cc}-r_{c} a_{1} & -r_{c} m c_{1}+r_{c} m c_{1} \\ 0 & 0\end{array}\right]+\left[\begin{array}{cc}0 & c_{M} \\ 0 & c_{S}\end{array}\right]=\left[\begin{array}{cc}-r_{c} a_{1} & c_{M} \\ 0 & c_{S}\end{array}\right]=\left[\begin{array}{cc}-\psi_{2}(c) a_{1} & c_{M} \\ 0 & c_{S}\end{array}\right]$. So, $c_{R}=-\psi_{2}(c) a_{1}$.

On the other hand, $\delta\left(c e_{22}\right)=\delta\left(e_{22} c e_{22}\right)$ implies that $c_{S}=-c_{1} c$. Thus $\delta\left(c e_{22}\right)=\left[\begin{array}{cc}-\psi_{2}(c) a_{1} & c_{M} \\ 0 & -c_{1} c\end{array}\right]$. 
Therefore we can define $h: S \rightarrow M$ by $h(c)=c_{M}$ where $\delta\left(c e_{22}\right)=$ $\left[\begin{array}{cc}-\psi_{2}(c) a_{1} & c_{M} \\ 0 & -c_{1} c\end{array}\right]$

Now $\forall a \in R, b \in M, c \in S, \delta\left(\left[\begin{array}{cc}0 & a b \\ 0 & 0\end{array}\right]\right)=\delta\left(a e_{11}\left[\begin{array}{ll}0 & b \\ 0 & 0\end{array}\right]\right)=\alpha_{2}\left(a e_{11}\right)$ $\delta\left(\left[\begin{array}{ll}0 & b \\ 0 & 0\end{array}\right]\right)+\delta\left(a e_{11}\right)\left[\begin{array}{ll}0 & b \\ 0 & 0\end{array}\right]=\left[\begin{array}{cc}0 & m \phi_{2}(a) \\ 0 & \phi_{2}(a)\end{array}\right]\left[\begin{array}{cc}0 & m g(b)+a_{1} b \\ 0 & g(b)\end{array}\right]+$ $\left[\begin{array}{cc}a_{1} a & m \phi_{2}(a) c_{1} \\ 0 & \phi_{2}(a) c_{1}\end{array}\right]\left[\begin{array}{ll}0 & b \\ 0 & 0\end{array}\right]=\left[\begin{array}{cc}0 & m \phi_{2}(a) g(b) \\ 0 & \phi_{2}(a) g(b)\end{array}\right]+\left[\begin{array}{cc}0 & a_{1} a b \\ 0 & 0\end{array}\right]$

$=\quad\left[\begin{array}{cc}0 & m \phi_{2}(a) g(b)+a_{1} a b \\ 0 & \phi_{2}(a) g(b)\end{array}\right] \quad$ and

$\left[\begin{array}{cc}0 & m g(a b)+a_{1} a b \\ 0 & g(a b)\end{array}\right]$. Thus $g(a b)=\phi_{2}(a) g(b)$

Similarly, $\delta\left(\left[\begin{array}{cc}0 & b c \\ 0 & 0\end{array}\right]\right)=\delta\left(\left[\begin{array}{ll}0 & b \\ 0 & 0\end{array}\right] c e_{22}\right)$ implies that $g(b c)=g(b) c$.

Moreover, $\delta\left(c^{2} e_{22}\right)=\delta\left(c e_{22} s e_{22}\right)=\alpha_{2}\left(c e_{22}\right) \delta\left(s e_{22}\right)+\delta\left(c e_{22}\right) s e_{22}$ $=\left[\begin{array}{cc}\psi_{2}(c) & -\psi_{2}(c) m \\ 0 & \end{array}\right]\left[\begin{array}{cc}-\psi_{2}(s) a_{1} & h(s) \\ 0 & -c_{1} s\end{array}\right]+\left[\begin{array}{cc}-\psi_{2}(c) a_{1} & h(c) \\ 0 & -c_{1} c\end{array}\right] s e_{22}$ $=\left[\begin{array}{cc}-\psi_{2}(c) \psi_{2}(s) a_{1} & \psi_{2}(c) h(s)+\psi_{2}(c) m c_{1} s \\ 0 & 0\end{array}\right]+\left[\begin{array}{cc}0 & h(c) s \\ 0 & -c_{1} c s\end{array}\right]$ $=\left[\begin{array}{cc}-\psi_{2}(c) \psi_{2}(s) a_{1} & \psi_{2}(c) h(s)+\psi_{2}(c) m c_{1} s+h(c) s \\ 0 & -c_{1} c s\end{array}\right]$ and $\delta\left[\begin{array}{cc}0 & 0 \\ 0 & c s\end{array}\right]=$ $\left[\begin{array}{cc}-\psi_{2}(c s) a_{1} & h(c s) \\ 0 & -c_{1} c s\end{array}\right]$. Thus $h(c s)=\psi_{2}(c) h(s)+h(c) s+\psi_{2}(c) m c_{1} s$.

Conversely, let $\delta\left(\left[\begin{array}{ll}a & b \\ 0 & c\end{array}\right]\right)=\left[\begin{array}{cc}a_{1} a-\psi_{2}(c) a_{1} & B \\ 0 & \phi_{2}(a) c_{1}+g(b)-c_{1} c\end{array}\right]$, where $B=m \phi_{2}(a) c_{1}+a_{1} b+m g(b)+h(c)$. Then we can check $\delta$ is an $\alpha_{2}-$ derivation by a similar proof of Theorem 3.1.

Theorem 3.5. Let $\alpha_{3}: T \rightarrow T$ be a Type III homomorphism. Then $\delta: T \rightarrow T$ is an $\alpha_{3}$-derivation if and only if there exist

(i) $f: R \rightarrow R$ is a $\phi_{3}$-derivation,

(ii) $g: M \rightarrow S$ is an additive map satisfying $\forall a \in R, b \in M, c \in S, g(a b)=$ $\psi_{3}(a) g(b)$ and $g(b c)=g(b) c$ and

(iii) $h: M \rightarrow M$ is an additive map satisfying $\forall a \in R, b \in M, c \in S, h(a b)=$ $\phi_{3}(a) h(b)+\theta_{3}(a) g(b)+f(a) b$ and $h(b c)=h(b) c$ such that for some $b_{1} \in M, c_{1} \in$ $S$,

$$
\delta\left(\left[\begin{array}{ll}
a & b \\
0 & c
\end{array}\right]\right)=\left[\begin{array}{cc}
f(a) & \phi_{3}(a) b_{1}+\theta_{3}(a) c_{1}+h(b)-b_{1} c \\
0 & \psi_{3}(a) c_{1}+g(b)-c_{1} c
\end{array}\right]
$$

where $\phi_{3}, \psi_{3}, \theta_{3}$ are maps given in Type III homomorphisms in Section 2. 
Sketch of proof. Let $\delta\left(e_{11}\right)=\left[\begin{array}{cc}a_{1} & b_{1} \\ 0 & c_{1}\end{array}\right]$. Then $\delta\left(e_{11}\right)=\left[\begin{array}{cc}0 & b_{1} \\ 0 & c_{1}\end{array}\right]$ and $\delta\left(e_{22}\right)=\left[\begin{array}{ll}0 & -b_{1} \\ 0 & -c_{1}\end{array}\right]$.

Let $\delta\left(a e_{11}\right)=\left[\begin{array}{cc}a_{R} & a_{M} \\ 0 & a_{S}\end{array}\right]$. Then $\delta\left(a e_{11}\right)=\left[\begin{array}{cc}a_{R} & \phi_{3}(a) b_{1}+\theta_{3}(a) c_{1} \\ 0 & \psi_{3}(a) c_{1}\end{array}\right]$. Thus $a_{S}=\psi_{3}(a) c_{1}$ and $a_{M}=\phi_{3}(a) b_{1}+\theta_{3}(a) c_{1}$.

Define $f: R \rightarrow R$ by $f(a)=a_{R}$. Then $f$ is an $\phi_{3}$-derivation. $b_{R}=0$.

Let $\delta\left(\left[\begin{array}{ll}0 & b \\ 0 & 0\end{array}\right]\right)=\left[\begin{array}{cc}b_{R} & b_{M} \\ 0 & b_{S}\end{array}\right]$. Then $\delta\left(\left[\begin{array}{ll}0 & b \\ 0 & 0\end{array}\right]\right)=\left[\begin{array}{cc}0 & b_{M} \\ 0 & b_{S}\end{array}\right]$. So,

Define $g: M \rightarrow S$ by $g(b)=b_{S}$. Then $g$ satisfies $\forall a \in R, b \in M, c \in$ $S, g(a b)=\psi_{3}(a) g(b)$ and $g(b c)=g(b) c$.

Define $h: M \rightarrow M$ by $h(b)=b_{M}$. Then $h$ satisfies $\forall a \in R, b \in M, c \in$ $S, h(a b)=\phi_{3}(a) h(b)+\theta_{3}(a) g(b)+f(a) b$ and $h(b c)=h(b) c$.

Let $\delta\left(c e_{22}\right)=\left[\begin{array}{cc}c_{R} & c_{M} \\ 0 & c_{S}\end{array}\right]$. Then $\delta\left(c e_{22}\right)=\left[\begin{array}{cc}0 & -b_{1} c \\ 0 & -c_{1} c\end{array}\right]$. This means

$$
\delta\left(\left[\begin{array}{ll}
a & b \\
0 & c
\end{array}\right]\right)=\left[\begin{array}{cc}
f(a) & \phi_{3}(a) b_{1}+\theta_{3}(a) c_{1}+h(b)-b_{1} c \\
0 & \psi_{3}(a) c_{1}+g(b)-c_{1} c
\end{array}\right] .
$$

Conversely, let $\delta\left(\left[\begin{array}{ll}a & b \\ 0 & c\end{array}\right]\right)=\left[\begin{array}{cc}f(a) & \phi_{3}(a) b_{1}+\theta_{3}(a) c_{1}+h(b)-b_{1} c \\ 0 & \psi_{3}(a) c_{1}+g(b)-c_{1} c\end{array}\right]$.

Then we can check $\delta$ is an $\alpha_{3}$-derivation by a similar proof of Theorem 3.1.

Theorem 3.6. Let $\alpha_{4}: T \rightarrow T$ be a Type IV homomorphism. Then $\delta:$ $T \rightarrow T$ is an $\alpha_{4}$-derivation if and only if there exist

(i) $g: S \rightarrow S$ is a $\psi_{4}$-derivation and

(ii) $h: S \rightarrow M$ is an additive map satisfying $\forall c, s \in S, h(c s)=\phi_{4}(c) h(s)+$ $\theta_{4}(c) g(s)+h(c) s$ such that for some $a_{1} \in R$,

$$
\delta\left(\left[\begin{array}{ll}
a & b \\
0 & c
\end{array}\right]\right)=\left[\begin{array}{cc}
a_{1} a-\phi_{4}(c) a_{1} & a_{1} b+h(c) \\
0 & g(c)
\end{array}\right]
$$

for some $a_{1} \in R$, where $\phi_{4}, \psi_{4}, \theta_{4}$ are maps given in Type IV homomorphisms in Section 2.

Proof. Since the proof is similar to above theorems, we omit.

\section{References}

[1] P. N. Anh, L. Van Wyk, Automomorphism groups of generalized triangular matrix rings, Linear Algebra Appl., 434(2011), 1018-1026.

[2] H. Ghahramani, Skew polynomial rings of formal triangular matrix rings, J.Algebra, 349, (2012), 201-216.

[3] H. Ghahramani, A. Moussavi, Differential polynomial rings of triangular matrix rings, Bulletin of the Iranian Mathematical Society, 34(2), (2008), 71-96. 
[4] M. N. Ghosseiri, The structure of $(\alpha, \beta)$-derivations of triangular rings, Iranian Journal of Science \& Techonology, Transaction A, 29(A3), (2005), 507-514.

[5] R. Khazal, S. Dascalescu, L. Van Wyk, Isomomorphism of generalized triangular matrixrings and recovery of tiles, Internat. J. Math. Sci., 2003(9), (2003), 533-538.

JANG Ho ChUN

DEPT. OF MATH.

YEUNGNAM UNIVERSITY

KYONGSAN

712-749

KOREA

E-mail address: jhchun@yu.ac.kr

JUNE WON PARK

Dept. of Undeclared Majors

KYUNGIL UNIVERSITY

KYONGSAN

712-701

KOREA

E-mail address: jwpark@kiu.ac.kr 